\title{
HIGH FREQUENCY ECR ION SOURCE (60 GHZ) IN PREGLOW MODE FOR BUNCHING OF BETA-BEAM ISOTOPES
}

\author{
T.Thuillier ${ }^{1}$, L. Latrasse, T. Lamy, C. Fourel, J. Giraud, \\ Laboratoire de Physique Subatomique et de Cosmologie, CNRS/IN2P3-UJF-INP Grenoble, \\ 53, rue des Martyrs 38026 Grenoble CEDEX, France \\ E-mail: thomas.thuillier@lpsc.in2p3.fr \\ C. Trophime, P. Sala, J. Dumas, F. Debray \\ Laboratoire des Champs Magnétiques Intenses, CNRS \\ 25 rue des Martyrs, B.P. 166, 38042 Grenoble CEDEX 9 France
}

The efficient production of short pulses of radioactive ion beams is one of the key points of the Beta-Beam project. A strong R\&D effort in the field of ion sources is required to reach this challenging objective. A summary of the preliminary studies performed during the 6th PCRD is proposed. Ion source main specifications are summarised. A first $60 \mathrm{GHz}$ ECR Ion Source prototype using room temperature coils is presented. The 3D magnetic field structure, along with the mechanical design status is presented. An experimental test using an aluminium coil prototype is presented, showing good agreement with simulation and validating the design. The Prototype is expected to be completed in the early 2009. Experiments at $60 \mathrm{GHz}$ may start in 2010.

10th International Workshop on Neutrino Factories, Super beams and Beta beams

Valencia, Spain

30 June - 05 July, 2008

\footnotetext{
1 Speaker
} 


\section{Summary of Preglow studies}

Experiments have been performed on a modified version of the PHOENIX source [1] named PHOENIX V2. Details of the experimental setup are reported in [2]. At the beginning of the RF pulse, for some tunings, a fast and intense extracted beam was observed, associated to a pressure decrease in the source. This peak was named Preglow (PGW) $[2,3]$ in analogy with the afterglow peak which occurs at the microwave pulse end. The evolution of $\mathrm{He}$ and Ne PGWs has been studied as a function of the following parameters: RF frequency (18 and $28 \mathrm{GHz}$ ) and power, buffer gas nature and pressure and plasma electrode hole diameter. To evaluate efficiencies, a Leybold calibrated leak TL6 $\left(5 \times 10^{-6}\right.$ mbar.1. $\left.\mathrm{s}^{-1}\right)$ has been used to inject either ${ }^{3} \mathrm{He}$ or ${ }^{22} \mathrm{Ne}$ isotopes. The technique used to analyze the experimental ionic pulses is described in [3].

Table 1. PGW characteristics of $\mathrm{He}+$ and $\mathrm{Ne} 2+$ peaks at 18 and $18 \mathrm{GHz}$.

\begin{tabular}{|l|l|l|l|l|}
\hline & $\mathrm{He}^{+} 18 \mathrm{GHz}$ & $\mathrm{He}^{+} 28 \mathrm{GHz}$ & $\mathrm{Ne}^{2+} 18 \mathrm{GHz}$ & $\mathrm{Ne}^{2+} 28 \mathrm{GHz}$ \\
\hline FWHM $(\mu \mathrm{s})$ & $600-1200$ & $100-300$ & 900 & 350 \\
\hline Bunching efficiency & $\sim 5-10 \%$ & $\sim 1-3 \%$ & $\sim 3 \%$ & $\sim 5 \%$ \\
\hline
\end{tabular}

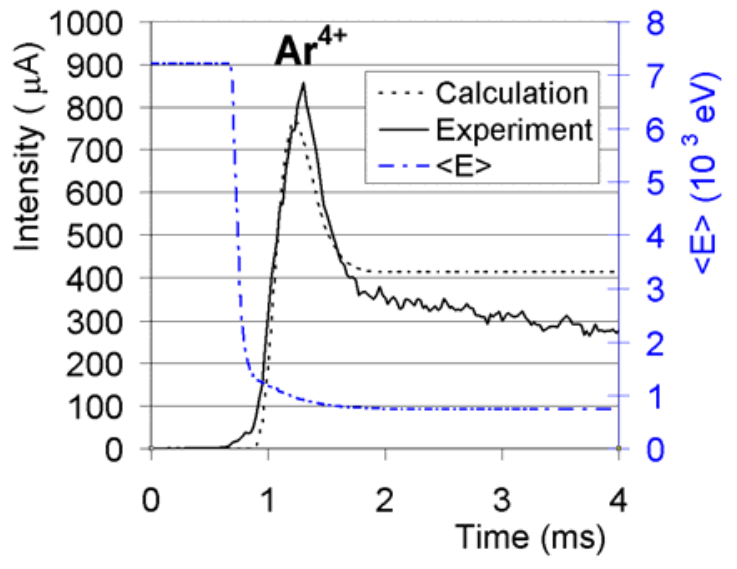

Figure 1. Experimental $\mathrm{Ar}^{4+}$ pulse and its numerical fits, along with $<\mathrm{E}>(\mathrm{t})$.
Table 1 summarizes examples of PGW peaks characteristics obtained for $\mathrm{He}^{+}$and for a $\mathrm{Ne}^{2+}$ for 18 and $28 \mathrm{GHz}$ microwave frequencies. It can be noticed that the Full Width Half Maximum (FWHM) of the PGW peaks decreases with the microwave frequency. This point is of importance since it incites to increase RF frequency to reduce FWHM. The $\mathrm{Ne}^{2+}$ global peak ionization efficiency also increases with the RF frequency from 3 to $5 \%$. On the contrary, a lower He efficiency was measured for $28 \mathrm{GHz}$ with respect to 18 GHz. This effect is mainly due to the too low magnetic confinement of PHOENIX V2

at $28 \mathrm{GHz}$, and to the ion extraction space charge limitation. Indeed, the best results of $3 \%$ were obtained with a high pressure in the source and a high extracted ionic current level which could not be properly accelerated through the beam line. This limitation could be avoided with a high voltage extraction system (typically $100 \mathrm{kV}$ ). With a better extraction system, He efficiency is expected to be higher at $28 \mathrm{GHz}$ than at $18 \mathrm{GHz}$ heating frequency. Another important fact is observed: the lower the plasma chamber volume, the lower amount of additional buffer gas is necessary to sustain the plasma and the lower the total extracted ionic current. These points are discussed further in the text.

A theoretical 0 dimension plasma model, described in [4], was developed by the Institute of Applied Physics of Nizhniy Novgorod (Russia) in order to describe the PGW mode. This model is based on the 18 and $28 \mathrm{GHz}$ experiments and it enables to simulate $60 \mathrm{GHz}$ plasmas. 
Figure 1 represents the experimental and simulated $\mathrm{Ar}^{4+}$ PGW peaks, along with the calculated electron energy averaged over the electron energy distribution function $(<E>(t))$. The microwave frequency influence on the FWHM and on the maximum intensity has been studied by simulation. As observed in experiments, the peak FWHM is expected to decrease with the ECR frequency increase. At a microwave frequency of $60 \mathrm{GHz}$, the FWHM should be of the order of $100 \mu$ s, and the peak currents are expected to increase by a factor 3 .

\section{Summary of specifications for the Beta Beam pulsed ion source}

According to the beta beam baseline scenario [5], the ion source should bunch the beam with a pulse duration as short as possible $(\sim 50-100 \mu \mathrm{s})$ with a pulse repetition rate in the range $\sim 10-25 \mathrm{~Hz}$. The expected radioactive atoms flux ${ }^{18} \mathrm{Ne}$ and ${ }^{6} \mathrm{He}$ from the targets are $\sim 5.10^{13} / \mathrm{s}$. This flux corresponds to $\sim 8 \mathrm{p} \mu \mathrm{A}$ continuous working $(\mathrm{CW})$ current. Such ionic intensity is very easy to extract from a classical ECRIS in CW mode. The highest peak current, derived from the former duty factor is $\sim 16 \mathrm{pmA}$. Moreover, an unknown amount of other gas species will diffuse from the target; these gases will also be transformed into current. The total extracted current from the source is thus expected to be high. In order to ensure high current, fast ionization and high production efficiency, the Beta Beam pulsed ion source requires high density plasma. A $4^{\text {th }}$ generation ECRIS heated at $60 \mathrm{GHz}$ is the best option. The volume should be as small as possible in order to increase the efficiency. A high voltage $(100 \mathrm{kV})$ is required to extract the high currents.

\section{Design of a $60 \mathrm{GHz}$ ion source in cusp configuration}

In order to investigate the topic, LPSC team decided to start an ambitious $60 \mathrm{GHz}$ R\&D program. The goal is to build several prototypes of $60 \mathrm{GHz}$ innovative magnetic structures and test them in pulsed mode. The structure foreseen may be as simple as a single field gradient to a complex minimum- $|\mathrm{B}|$ structure. The development of several operational superconducting technologies at $60 \mathrm{GHz}$ is not realistic since the feasibility of such compact magnetic structures is not proven, and since it is time consuming and very expensive. The collaboration with the Grenoble High Magnetic Field Laboratory (GHMFL) allows making easily $60 \mathrm{GHz}$ ECRIS R\&D for an affordable price and reduced design time. GHMFL is equipped with a set of 20 MW/35 T resistive coils available for fundamental physics studies. The original idea consists in developing $60 \mathrm{GHz}$ prototype magnetic coils using the helix coil resistive technology [6] invented at GHMFL, and test them on site with a new dedicated ECRIS test bench. The $60 \mathrm{GHz}$ prototypes will be dimensioned to comply with the GHMFL electrical power and water cooling systems. Moreover, this technology is usable in a highly radioactive environment, since the magnetic structure is mainly composed of copper, steel and water. As a first step, LPSC and GHMFL decided to design an axi-symmetric MHD stable magnetic structure: a cusp. The initial design specifications include the following magnetic properties: a closed $2.1 \mathrm{~T} 60 \mathrm{GHz}$ ECR zone, a 4 Tesla radial mirror; $6 \mathrm{~T}$ at the injection; $3 \mathrm{~T}$ at the extraction and a $10 \mathrm{~cm}$ axial mirror length. Field lines going through the ECR zone must be connected to the magnetic mirrors without intercepting the plasma chamber wall. 


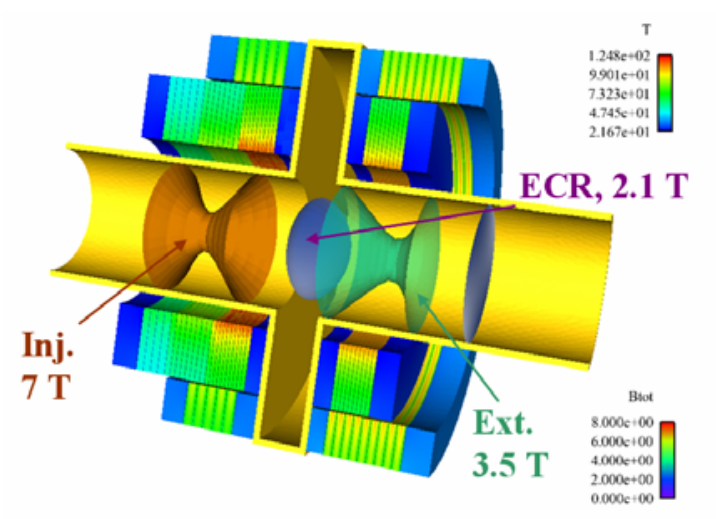

Figure 4: 3D simulation results.

A 2D simulation was first performed using both RADIA [7] and Getdp (software developed at Liege University), specially adapted to the helix technique [8]. A flexible solution was found with a set of 4 radially cooled helices coils. The simulation continued with 3D calculations performed with another code still based on Getdp [9]. It includes thermal analysis (helices water cooling) and exact helices geometry (width of the electro erosion slit). The helices were designed with CATIA and then meshed by SAMCEF Field. The helices conductivity is $90 \%$ of the International Annealed Copper Standard (IACS). 3D simulations gave results in good agreement with the $2 \mathrm{D}$ ones. A sectional view of the magnetic structure can be seen in figure 4. There, the iso-B surface of $7 \mathrm{~T}$ at the injection, the iso- $\mathrm{B}$ surface of $3.5 \mathrm{~T}$ at the extraction and the iso-B of $2.1 \mathrm{~T}$ (ECR zone) are represented in the plasma chamber along with the temperature in the helices. For more visibility, the mirror surface of $4.9 \mathrm{~T}$ in the shoulder of the plasma chamber is not represented. These values are above specifications, so more tuning flexibility will be available for the ion source.

An aluminium helix prototype has been machined to experimentally validate the accuracy of the calculations (see figure 5(a)). Figure 5(b) represents a comparison between the calculated and the measured axial magnetic field along the coil axis at low current density (144 A injected). $\mathrm{z}=0 \mathrm{~mm}$ is the beginning of the helix on the thin pitch side. The difference is only of $3 \%$ at the peak value and both curves have the same magnetic centre. The test validates the simulation and allows to start the construction of the magnetic structure. A sectional view of the 3D mechanical design is displayed on figure 5(c).
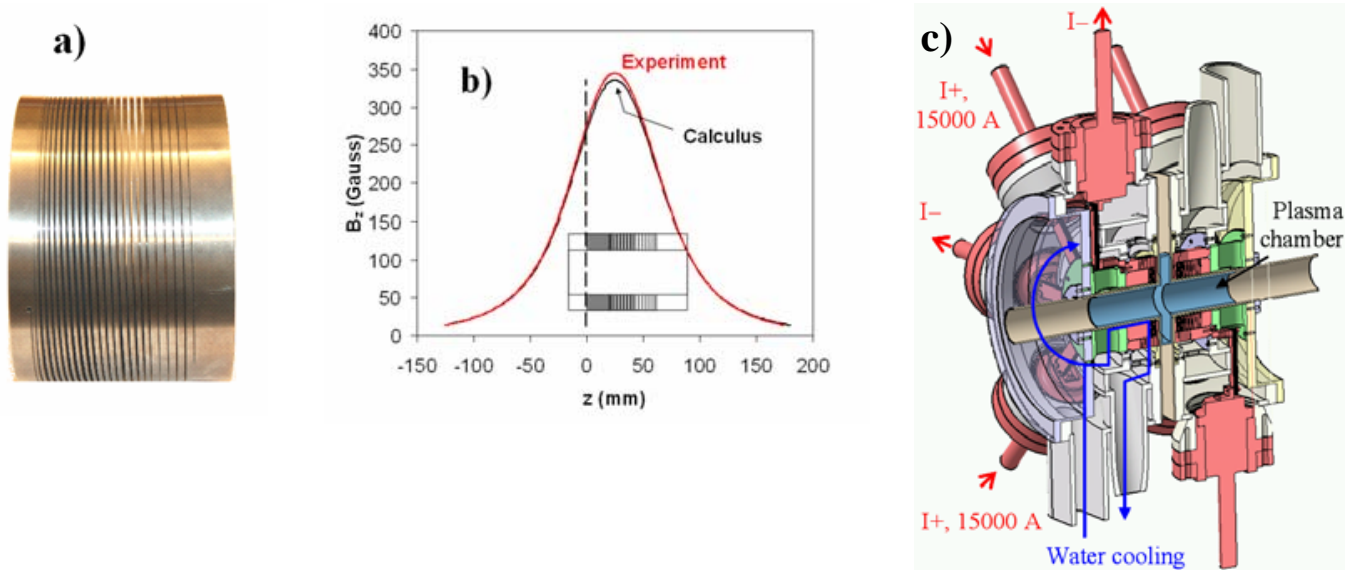

Figure5. a) Aluminium coil prototype, b) Experimental and simulated coil magnetic field on axis, c) 3D mechanical design.

\subsection{Engineering design}

The current intensity in the cusp is $30000 \mathrm{~A}$; the maximum current density reaches $640 \mathrm{~A} / \mathrm{mm}^{2}$ on the internal radius of the coils, where the helix pitch is only $2 \mathrm{~mm}$. The 
maximum electrical power needed is estimated to be about $5 \mathrm{MW}$, depending on the final coils resistivity, so the structure is actively cooled with de-ionized water flow. The inlet water pressure is $2.7 \mathrm{MPa}$ (27 bar), while the outlet one is $0.4 \mathrm{MPa}$ (4 bar). The average water temperature increase is $20^{\circ} \mathrm{C}$. The maximum water flow rate is $\sim 20 \mathrm{l} / \mathrm{s}$. The water speed in the radial helices slit ranges within 30 to $35 \mathrm{~m} / \mathrm{s}$ providing a convection heat transfer coefficient $\mathrm{h} \sim 150 \mathrm{~kW} / \mathrm{m}^{2} /{ }^{\circ} \mathrm{C}$. The average coils temperature varies from 70 to $95{ }^{\circ} \mathrm{C}$, while the peak temperature locally reaches $125{ }^{\circ} \mathrm{C}$. Pessimist calculations have been performed using a conductivity of $80 \%$ IACS and $\mathrm{h} \sim 120 \mathrm{~kW} / \mathrm{m}^{2} /{ }^{\circ} \mathrm{C}$ for each coil. In this case, the average temperature of the coils is between 80 and $115^{\circ} \mathrm{C}$, the peak temperature reaches $150{ }^{\circ} \mathrm{C}$ and the power needed is $6.5 \mathrm{MW}$. In these conditions, the maximum hoop stress in the coils is $\sigma \sim 280$ $\mathrm{MPa}$, far below the copper alloy limit of elasticity (360 MPa). At full current, the two sets of coils repel each other with a force of $600 \mathrm{kN}$.

\subsection{Planning}

This first $60 \mathrm{GHz}$ magnetic structure (helices coils in their tanks, electrical and water cooling environment) should be available at the beginning of 2009. The $60 \mathrm{GHz}$ Gyrotron is expected at best for the end of 2009. First experiments of the prototype at a $28 \mathrm{GHz} \mathrm{ECR}$ frequency should be done in 2009. The first pulsed beam at $60 \mathrm{GHz}$ is expected in 2010 .

\section{References}

[1] T. Thuillier, J.L. Bouly, J.C. Curdy, E. Froidefond, T. Lamy, C. Peaucelle, P. Sole, P. Sortais, J.L. Vieux-Rochaz, D. Voulot, High Intensity Ion Beams Prospects for Accelerators with PHOENIX 28 GHz, in Proc. 8th European Particle Accelerator Conf., Paris, 2002, pp. 1744-1746.]

[2] T. Thuillier, T. Lamy, L. Latrasse, I. V. Izotov, A. V. Sidorov, V. A. Skalyga, V. G. Zorin, M. Marie-Jeanne, Study of pulsed electron cyclotron resonance ion source plasma near breakdown: The Preglow, Rev. Sci. Instrum., Vol. 79(2), 02A314-02A316, Feb. 2008.

[3] P. Sortais, J.L. Bouly, J.-C. Curdy, T. Lamy, P. Sole, T. Thuillier, J.L. Vieux-Rochaz, D. Voulot, ECRIS development for stable and radioactive pulsed beams, Rev. Sci. Instrum., Vol. 75(5), pp. 1610-1612, May 2004.

[4] I.V. Izotov, A.V. Sidorov, V.A. Skalyga, V.G. Zorin, T. Lamy, L. Latrasse, T. Thuillier, Experimental and theoretical investigation of the Preglow in ECRIS, IEEE Transactions on Plasma Science 36/4 (2008) 1494-1501

[5] http://cern.ch/beta-beam/

[6] P. Rub and W. Joss 1996 A new type of radially cooled helices designed for a 25 T magnet, IEEE Transactions on Magnetics 322570 - 3 .

[7] O. Chubar, P. Elleaume and J. Chavanne 1998 A three-dimensional magnetostatics computer code for insertion devices, J. Synchrotron Rad. 5481 - 4

[8] C. Trophime, K. Egorov, F. Debray, W. Joss and G. Aubert 2002 Magnet Calculations at the Grenoble High Magnetic Field Laboratory IEEE Trans. Appl. Superconduct. 121483 - 7.

[9] C. Trophime, S. Krämer and G. Aubert 2006 Magnetic Field Homogeneity Optimization of the Giga-NMR Resistive Insert IEEE Trans. Appl. Superconduct. 161509 - 12 